\title{
La mirada de los estudiantes sobre el desempeño de sus profesores
}

\section{A student look into their Professor"s Academic Performance}

Mtra. Blanca Julia Ponce Cortés

\author{
"Reconocer la evaluación de la docencia \\ Como objeto de estudio, \\ es generarle un espacio de análisis y reflexión \\ en el campo de la educación" \\ Javier Loredo Enríquez
}

\section{Resumen}

Introducción: A partir del estudio derivado del proyecto de "Evaluación del desempeño docente de los profesores de la Escuela Nacional de Enfermería y Obstetricia. Un enfoque formativo", se presenta este trabajo que hace énfasis en el desarrollo y el mejoramiento del proceso educativo, dándole un sentido estrictamente constructivo y propositivo. Objetivo: Generar la reflexión sobre la urgente necesidad de nuevas propuestas educativas conducentes a mejorar la formación de los profesionales de la enfermería. Desarrollo: En el contexto del análisis sobre la percepción de los estudiantes del desempeño docente de sus profesores en los procesos de evaluación realizados en la ENEO, se enuncian un conjunto de reflexiones a la luz de una nueva cultura docente que contribuya a que los alumnos aprendan a aprender, a plantear y resolver problemas, a pensar en forma crítica y a ser creativos. Conclusiones: La información sobre el desempeño de la práctica docente de los profesores es sólo un aspecto del proceso evaluativo. Su riqueza y, a la vez su mayor reto, consiste en la reflexión, interpretación, juicios y acciones a considerar en las instituciones educativas que se reflejen en el hacer docente

Palabras

Clave:

Evaluación, evaluación formativa, docencia, evaluación de la práctica docente.

- Profesor de Carrera Asociado "C" de Tiempo completo.

Correspondencia: blancajulia01@hotmail.com

RECIBIDO 3 DE NOVIEMBRE 2010

ENVIADO 17 DE NOVIEMBRE 2010

ACEPTAdo 28 DE FEBRERO 2011 


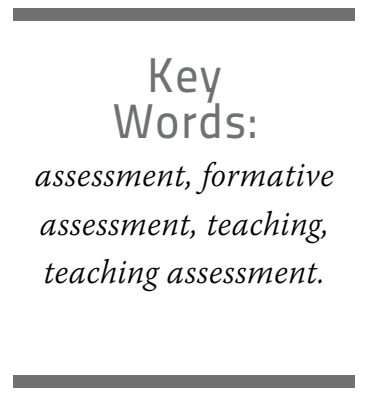

Abstract

Introduction: Constructively and positively biased, and taking in consideration the Project "ENEO-Professors Academic Performance Assessment: A formative Focus", we conducted a study related to the development and improvement of nursing education. Objective: To generate insights related to the urgency of new educational proposals which could help improve the preparation of the coming nursing professionals. Development: Within the framework of ENEO student's perceptions of their professors' academic performance, we outline a series of insights which could contribute to the students'learning to learn, problem solving, and critical and creative thinking forming processes. Conclusion: In order to improve their nursing curriculum, education instructions musk integrally assess their professors' academic performance.

\section{INTRODUCCIÓN}

La evaluación en su concepción más general implica emitir un juicio de valor relativo a los atributos del sujeto u objeto sobre el que se efectúa dicho proceso, en este sentido la pauta de significación desde donde se construye la evaluación es un terreno controversial que por principio no puede ser hegemónico, por tal razón la evaluación educativa es compleja, difícil de ejecutar e interpretar. Sin embargo, el valor de la información que se obtiene a través de ella posibilita tomar decisiones pertinentes para orientar o reorientar las estrategias que contribuyan a alcanzar los más trascendentes fines educativos.

La palabra evaluación frecuentemente genera más resistencias y temores, reacciones explicables por que a estado asociada, especialmente en el campo educativo, con los procesos de calificación, clasificación, exclusión y castigo, por citar algunos. Estas resistencias y temores son producidos, la mayoría de las veces, porque los procesos de evaluación son impuestos jerárquicamente y al margen de los sujetos evaluados en los que su único fin es juzgar el comportamiento de los involucrados.

La evaluación docente debe concebirse como un servicio a quienes participan en la educación, con un sentido esencialmente constructivo y propositivo en la que todos los involucrados tengan oportunidad de participar y como consecuencia mejorar su desempeño en el aula. Las universidades tienen el compromiso social de formar profesionistas útiles a la sociedad, en el caso de la ENEO; la disciplina de enfermería contribuye a resolver los problemas de salud del país. La naturaleza de las funciones y actividades de las instituciones universitarias, reclaman la existencia de procesos internos y externos de evaluación como procedimientos necesarios para garantizar la pertinencia, eficacia y eficiencia de los mismos.

En este contexto destaca como acción básica la evaluación de la práctica docente para identificar sus fortalezas y debilidades, con el propósito de diseñar procesos de mejora continua cuya estrate- gia fundamental sea la formación docente.

\section{DesarRollo}

\section{Los procesos de evaluación docente en la ENEO}

En 1993 se realizó un proceso de evaluación docente, bajo la responsabilidad del H. Consejo Técnico que diseñó y aplicó un instrumento de evaluación. Los resultados de carácter individual fueron entregados en un sobre cerrado a cada docente y se le sugirió acudir al Departamento de Pedagogía para que se le orientara sobre aspectos didácticos y metodológicos, con el propósito de mejorar su práctica. Cabe destacar que en este proceso se llevó a cabo la evaluación de los estudiante que participaban en las prácticas clínicas y comunitarias. Es interesante señalar que la sistematización de la información, se hizo manualmente con el apoyo de los docentes que fueron insaculados.

En los semestres 97-I, 97-II y 98-I se efectúo una Evaluación de la calidad docente cuyo cuestionario fue elaborado por un grupo de profesores en el que los reactivos fue- 
ron agrupados en cuatro categorías: Conocimiento de la materia, Capacidad didáctica, Cualidades para la evaluación y Actitudes personales. Entre los resultados obtenidos destacan las opiniones no satisfactorias en once aspectos de los cuales sobresalen las capacidades didácticas y las actitudes personales. De igual forma que en la anterior evaluación, los resultados fueron enviados a los docentes en sobres cerrados con una carta de invitación por parte de la Secretaria General para que reflexionaran sobre su práctica y solicitaran los recursos o apoyo para su mejora.

Los procesos de evaluación descritos se centraban en la decisión y voluntad del docente para modificar y mejorar su práctica, sin lograr los cambios esperados ya que desde la percepción de los alumnos los profesores continuaban con las tradicionales prácticas educativas sustentadas en la enseñanza y no en el aprendizaje.

Por lo antes expuesto fue necesario llevar a cabo un nuevo proceso de evaluación docente participativo y adecuado a los avances que sobre esta temática se habían desarrollado en otras instituciones del país y, como parte del mismo, surgiera un proceso de formación docente vinculado a las necesidades particulares de cada profesor o de los grupos colegiados.

En este contexto surge un nuevo proceso de evaluación de la práctica docente de los profesores de la ENEO con un enfoque de evaluación formativa a fin de favorecer en el docente la toma de conciencia y reflexión sobre su actuación en el aula. Desde el año 2005-2 hasta el 2009-1 se aplicó un cuestionario validado con el pro-

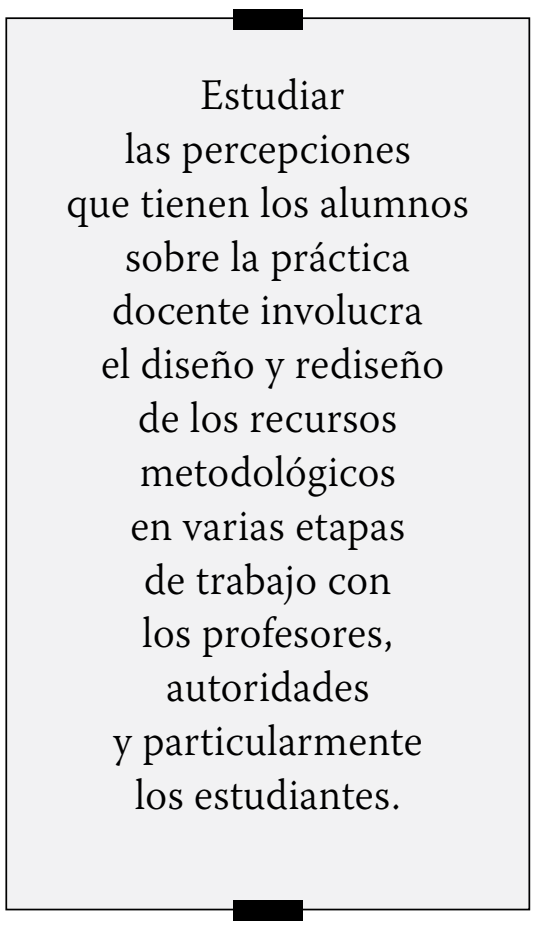

pósito de analizar e interpretar las opiniones vertidas por los alumnos sobre las prácticas docentes en el aula para reconocer actitudes y estilos de docencia que contribuyan al fortalecimiento de la formación profesional de los estudiantes de enfermería. Cabe indicar que para el procesamiento de la información se contó con el apoyo de recursos tecnológicos.

\section{ANÁLISIS E INTERPRETACIÓN DE LA OPINIÓN DE LOS ESTUDIANTES DE LA ENEO SOBRE LAS PRÁCTICAS DOCENTES EN EL AULA.}

La docencia como un aspecto central del proceso enseñanzaaprendizaje es susceptible de ser evaluada, de manera específica, con lo que el docente hace antes, durante y después de que ocurre el episodio didáctico: sus labores de planeación, impartición de clases revisión de trabajos, tareas y evaluación de los aprendizajes; con su pensamiento implícito y explícito en relación con la labor magisterial y la actuación derivada de ese pensamiento; con la actividad profesional que desarrolla como enseñante y la reflexión que despliega en torno a dicha actividad. ${ }^{1}$

La planeación de la docencia incluye lo que los profesores piensan lograr (objetivos) cómo piensan alcanzarlo (estrategias didácticas) y de qué manera pueden saber lo que lograron (estrategias de evaluación). Este análisis proporciona información valiosa para caracterizar lo que los profesores hacen en el aula y acercarnos a la comprensión de por qué lo hacen, por lo que, la evaluación de la práctica docente de los profesores practicada por los alumnos es una excelente fuente de información. ${ }^{2}$

De esta manera, la evaluación de la práctica docente de los profesores realizada por los alumnos se convierte en una excelente fuente de información pues son observadores naturales in situ, es decir los únicos que pueden observar las cosas que suceden en el aula con plena confianza y espontaneidad; son los usuarios y eso los convierte en los informantes de primera mano, a todas luces irremplazables, ${ }^{3}$ de aquí que la información obtenida represente una fuente de conocimiento acerca de las características de la práctica docente de los profesores de la ENEO y un lugar de gestación de mejoras educativas.

En consecuencia estudiar las percepciones que tienen los alumnos sobre la práctica docente involucra el diseño y rediseño de los recursos metodológicos en varias etapas de trabajo con los profesores, autoridades y particularmente los estudiantes. La construcción de un instrumento de evaluación docente debe ser acorde al contexto 
institucional donde se ejerce cierto estilo de docencia, siendo la referencia sustantiva los propósitos curriculares planteados en el Perfil de Egreso del Plan de Estudios de la Licenciatura en Enfermería y Obstetricia, que están orientados hacia una educación integral de los alumnos y hacia una práctica profesional centrada en el Cuidado Integral a las personas sanas o enfermas con una modalidad de trabajo interdependiente con otros profesionales de la salud e independiente con base en la valoración de necesidades de salud que no son atendidas por otros profesionales. ${ }^{4}$

En el caso de la Docencia en la ENEO y en el marco de las reflexiones hasta aquí vertidas, fueron tres las categoría que dieron estructura al último instrumento de evaluación docente utilizado: Metodología de Enseñanza Aprendizaje, Evaluación del Aprendizaje y Características Personales, con una escala valorativa de Siempre, Casi Siempre, Algunas Veces y Nunca.

Dichas categorías configuran en conjunto un perfil de docencia dinámico en el que prevalecen de manera singular preocupaciones afines entre los estudiantes de diversos semestres y años de estudio, siendo estas coincidencias el objeto de análisis.

En el caso de la categoría de metodología de la enseñanza que refiere actividades al interior del aula relacionadas con el desarrollo de las habilidades de análisis, síntesis, pensamiento crítico, capacidad para resolver y plantear nuevo problemas, búsqueda y manejo de información a través de diferentes medios, creatividad, desarrollo de la autonomía para el aprendizaje y la capacidad para el trabajo colabo- rativo, en opinión de los alumnos son mínimamente realizadas por los docentes. Lo cual implica que los estudiantes de la ENEO tengan dificultades en la transferencia del conocimiento aprendido a situaciones que la práctica de enfermería les demanda, además no se logra aceptablemente el desarrollo de actitudes y valores que les permitan establecer relaciones personales favorables sustentadas en la comprensión y respeto al otro, así como la disposición para trabajar en proyectos colectivos con otros profesionales.

En la categoría de Evaluación del Aprendizaje los resultados coinciden en indicar que los docentes a lo largo del semestre pocas veces realizan procesos de evaluación, lo cual obstaculiza la función retroalimentadora de la evaluación y en consecuencia la falta de promoción de la autoevaluación entre los alumnos, a grado tal que el estudiante escasamente puede explicar la relación entre su desempeño académico y la calificación obtenida.

Finalmente en la categoría Características Personales, los alumnos identifican que un grupo de docentes no inicia las clases en la hora indicada. El fenómeno de la impuntualidad docente pudiera tener variadas fuentes de explicación, sin embargo también conlleva serias contradicciones y graves con-

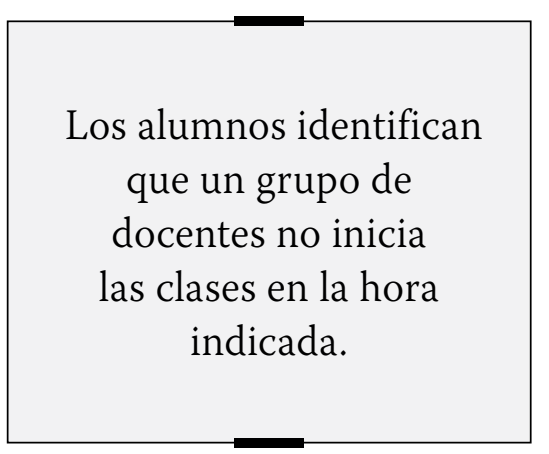

secuencias, por ejemplo cuando el docente expresa que el tiempo asignado para el abordaje del programa le es insuficiente y al no concluir los temas o los da por vistos o para que sean revisados sólo por los alumnos.

Como se puede apreciar la mirada de los alumnos sobre la docencia conduce a replantear más allá del discurso los mecanismos que logren consolidar el andamiaje para alcanzar la formación integral de los alumnos en la adquisición de información, el desarrollo de capacidades y el desarrollo de la subjetividad.

\section{Conclusiones}

Evaluar hace referencia a cualquier proceso en el que alguna o varias características de un alumno, de un grupo de estudiantes, de un ambiente educativo, de objetivos educativos, de materiales, de profesores, programas etc., reciben la atención del que evalúa, se analizan y se valoran sus características y condiciones en función de unos criterios o puntos de referencia para emitir un juicio que sea relevante para la educación, en este sentido, el aspecto central de evaluación es la valoración, es decir, emitir un juicio en referencia a un criterio. ${ }^{5}$

En las últimas décadas la evaluación de la docencia ha sido una preocupación importante de las instituciones de educación superior y ante tal preocupación nos preguntamos ¿Por qué evaluar la práctica docente de los profesores?

Una de las respuestas que nos surgen ante esta interrogante $y$ coincidiendo con Crispín, Román y Loyo ${ }^{6}$ es, porque el papel que se espera del profesor ha cambiado, porque los objetivos educativos 
se han transformado, antes se esperaba que el docente fuera capaz de impartir una buena cátedra y transmitir sus conocimientos de una manera clara, ahora se tiene la expectativa que el profesor diseñe estrategias para que los alumnos aprendan a aprender, a plantear y resolver problemas, a pensar en forma crítica y a ser creativos.

Actualmente nadie puede negar la importancia que se atribuye al desarrollo de las habilidades para "aprender a aprender" derivada no sólo de concepciones teóricas propias del ámbito educativo, sino de las demandas sociales que requieren la formación de individuos capaces de un mayor manejo autónomo de herramientas cognitivas, debido a los procesos de evolución y transformación de los conocimientos en general y de los tecnológicos en particular. ${ }^{7}$

La verdadera docencia cita Morán, ${ }^{8}$ son aquellas acciones en el aula que propician que el alumno se forje la necesidad de aprender por su cuenta, encontrando en el profesor un guía, un acompañante de travesía para llegar al conocimiento y en el grupo un espacio de encuentro, de intercambio, discusión y confrontación de ideas, buscando no tan sólo aprender sino que a través de una evaluación formativa, aprenda la forma de aprender, es decir, entender la evaluación como aprendizaje. Esta concepción de docencia conlleva un trabajo profundo desde diversos ángulos de la vida educativa.

Coincidimos con Quesada y Rubio $^{9}$ en que la práctica profesional de la enfermería se ha hecho más compleja debido a los avances científicos y tecnológicos así como por la especialización de los procesos asistenciales que exigen otras capacidades de la enfermera y del enfermero profesional además de su destreza técnica y su sentido humanista, pues ya no basta la información cuyo dominio es imposible, ahora se requiere saber buscar, procesar y comprender el conocimiento para enfrentar la solución de los problemas que competen al ejercicio profesional, por eso en el perfil de egreso se enfatizan las capacidades de razonamiento lógico, juicio crítico, creatividad y espíritu científico que posibilite la autonomía de los estudiantes, el desarrollo de valores y actitudes para establecer relaciones personales favorables.

Implica también establecer un cambio radical en la relación pedagógica que posibilite por un lado, diferentes formas de acceder al conocimiento, que lleven al estudiante a realizar actividades múltiples y variadas que le permitan poner en juego sus diversas capacidades, que los recobren como sujetos de aprendizaje, que se generen nuevos vínculos de relación permeados por la comunicación entre el docente y los estudiantes y una interacción permanente del grupo de aprendizaje. La docencia demanda mostrar entusiasmo por los logros del estudiante, interés por sus problemas y potencialidades respecto a sus propósitos de formación profesional y metas de desarrollo personal.

Una dimensión extra aúlica de la docencia que no se puede pasar por alto por su trascendencia, es el trabajo colectivo de los profesores cuyo espacio potencial para hacerlo lo constituyen las Academias con su principal objetivo de analizar, reflexionar, compartir, difundir y crear nuevos conocimientos tendientes a solucionar problemas de su práctica. Esta forma de concebir, actuar y reflexionar sobre ésta, sentaría las bases para una docencia colectiva que seguramente tendría mayor impacto en la formación de los estudiantes que aquella individual, secreta, aislada como la que aún predomina en las instituciones educativas.

La información sobre el desempeño de la práctica docente es sólo un aspecto del proceso evaluativo. $\mathrm{Su}$ riqueza y, a la vez su mayor reto, consiste en la reflexión, interpretación, juicios y acciones a considerar en las instituciones educativas.

Es indispensable continuar y fortalecer la cultura de la evaluación docente a partir de toda la experiencia que durante estos años se ha venido acumulando, también es necesario incorporar procesos de investigación descriptivos y explicativos que fundamenten el sentido de la actualización y formación docente acordes con el perfil de egreso de los estudiantes, los avances de la disciplina de Enfermería y de las teorías educativas. Hoy en día la evaluación representa un punto medular en todas aquellas tareas educativas en tanto que la información obtenida posibilita la toma de decisiones antes, durante o al término de las acciones emprendidas.

En el campo de la evaluación académica lo más frecuente es encontrar dos posiciones respecto a la función que debe cumplir: formativa o sumativa. La formativa está centrada en los procesos, buscando proporcionar información respecto a las actividades que se desarrollan a fin de retroalimentar el desempeño que se está evaluando. ${ }^{10}$ El énfasis de esta evaluación se pone en el desarrollo, en el mejoramiento del proceso, dándole un 
sentido estrictamente constructivo y propositivo. En cuanto a la evaluación sumativa se trata fundamentalmente de una "valoración del producto" o resultado. Lo importante es asignarle un valor numérico a lo que está juzgando, mismo que servirá para un proceso de toma de decisiones.

Ante todo el panorama planteado sobre la evaluación docente y su complejidad, es de gran importancia incorporar otra mirada, tan valiosa como la anterior, la de los profesores, pues reconociendo su pensamiento y las condiciones en que realizan su práctica, se podrá explicar por qué hacen o dejan de hacer ciertas cosas en la cotidianeidad de las aulas. ${ }^{11}$

\section{Referencias Bibliográficas}

1 Rueda M, Díaz Barriga F. Evaluación de la docencia. Perspectivas actuales. México: Paidós Educador; 2006. p. 17.

2 Loredo EJ (Coord). Evaluación de la práctica docente en educación superior. México: Porrúa, Universidad Anáhuac; 2000. p. XI.

3 Aguirre X, Botero I, Loredo J, Magaña L, Montano, et. al. Evaluación integral de la docencia: Una pro- puesta formativa y humanista. En: Loredo EJ (Coord). Evaluación de la práctica docente en la educación superior. México: Porrúa, Universidad Anáhuac; 2000: 1-35

4 ENEO, Plan de Estudios de la Licenciatura en Enfermería y Obstetricia. México: ENEO-UNAM; 2000.

5 Sacristán- Gimeno J. La evaluación en la enseñanza. En: Sacristán- Gimeno J, Pérez Gómez AI. Comprender y transformar la enseñanza. $4^{\mathrm{a}}$ ed. Madrid: Morata, 1996, p. 37.

6 Crispín ML, Romay ML, Loyo C. Vinculación de procesos evaluativos a la formación de docentes universitarios. En: Rueda M. y Díaz -Barriga F (Coord). Evaluación de la docencia. Perspectivas actuales. México: Paidós Educador: 2006. p. 341.

7 Celman S. ¿Es posible mejorar la evaluación y transformarla en herramienta de conocimiento? En: La evaluación de los aprendizajes en el debate didáctico contemporáneo. Argentina: Paidós; 1998. p.56.

8 Morán P. Hacia una evaluación cualitativa en el aula. Reencuentro. Análisis de problemas universita- rios. 2007; 48:9-19.9 Ques ada C, Rubio S. La función docente en la enseñanza clínica de enfermería. En Espinosa A, Rodríguez, S. (Compiladoras) Antología, Docencia en Enfermería. México: ENEO-SUA-UNAM; 2009. p. 110

10 Canales A. Evaluación educativa. La oportunidad y el desafío. Reencuentro. 2007; 48:40-46.

11 Monroy FM. Evaluación de la práctica educativa a través de la reflexión del pensamiento didáctico docente. En: Rueda M. Díaz Barriga F. (Coord) Evaluación de la docencia. Perspectivas actuales, México: Paidós Educador, 2000. p.p. $283-284$

\section{Agradecimientos:}

A la Licenciada Celia Ramírez Salinas por sus valiosos comentarios y sugerencias al manuscrito 